\title{
Molecular Imaging of Ischemia and Reperfusion in Vivo with Mitochondrial Autofluorescence
}

\author{
Hsueh-Han Lu, ${ }^{\dagger}{ }^{\S}$ Yao-Ming Wu, ${ }^{\ddagger}, \S$ Wei-Tien Chang, ${ }^{\ddagger}$ Teng Luo, ${ }^{\dagger}$ Yi-Cyun Yang, ${ }^{\dagger}$ Hung-Der Cho, ${ }^{\dagger}$ \\ and Ian Liau ${ }^{*}{ }^{\dagger}$ \\ ${ }^{\dagger}$ Department of Applied Chemistry and Institute of Molecular Science, National Chiao Tung University, Hsinchu 300, Taiwan \\ ${ }^{\ddagger}$ National Taiwan University Hospital and College of Medicine, National Taiwan University, Taipei 100, Taiwan
}

\section{Supporting Information}

ABSTRACT: Ischemia and reperfusion (IR) injury consti-
tutes a pivotal mechanism of tissue damage in pathological
conditions such as stroke, myocardial infarction, vascular
surgery, and organ transplant. Imaging or monitoring of the
change of an organ at a molecular level in real time during IR is
essential to improve our understanding of the underlying
pathophysiology and to guide therapeutic strategies. Herein,
we report molecular imaging of a rat model of hepatic IR with
the autofluorescence of mitochondrial flavins. We demonstrate
a revelation of the histological characteristics of a liver in vivo
with no exogenous stain and show that intravital autofluor-
escent images exhibited a distinctive spatiotemporal variation
during IR. The autofluorescence decayed rapidly from the

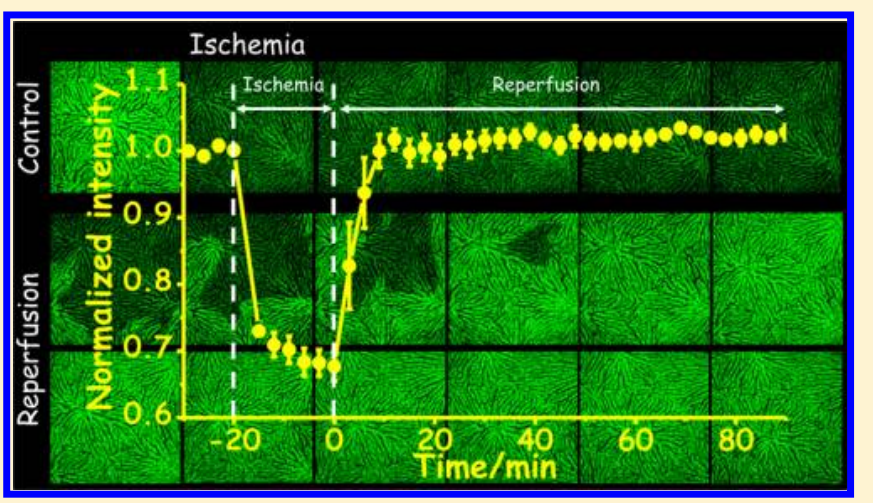
baseline immediately after 20-min ischemia (approximately 30\% decrease in $5 \mathrm{~min}$ ) but recovered gradually during reperfusion (to approximately $99 \%$ of the baseline $9 \mathrm{~min}$ after the onset of reperfusion). The autofluorescent images acquired during reperfusion correlated strongly with the reperfused blood flow. We show further that the autofluorescence was produced predominantly from mitochondria, and the distinctive autofluorescent variation during IR was mechanically linked to the altered balance between the flavins in the oxidized and reduced forms residing in the mitochondrial electron-transport chain. Our approach opens an unprecedented route to interrogate the deoxygenation and reoxygenation of mitochondria, the machinery central to the pathophysiology of IR injury, with great molecular specificity and spatiotemporal resolution and can be prospectively translated into a medical device capable of molecular imaging. We envisage that the realization thereof should shed new light on clinical diagnostics and therapeutic interventions targeting IR injuries of not only the liver but also other vital organs including the brain and heart.

\begin{abstract}
Tschemia and reperfusion (IR) underlie the pathophysiology of various critical diseases, including myocardial infarction and stroke. ${ }^{1}$ Injuries associated with IR might occur also to the heart during cardioplegic surgery or to the liver in hepatic transplant, so to compromise the outcome of patients. ${ }^{1}$ To improve our understanding of the pathogenesis of IR, it is important to be able to interrogate the molecular details of cellular changes during IR. From a clinical point of view, a development of a tool that allows timely and quantitative assessment of cells in vivo with great molecular specificity is equally important for the diagnosis of IR and the optimization of therapeutic strategies targeting IR.

Molecular imaging has emerged as a powerful analytical tool for both clinical diagnostics and biomedical research. ${ }^{2,3}$ Among molecular imaging of varied modality, optical imaging with fluorescence is of particular interest because of its inherent great sensitivity and specificity, and superb spatiotemporal resolution. ${ }^{4}$ By injecting fluorescent probes designed to respond to specific molecular events or environmental stresses, pathophysiological changes of cells or tissues underling IR can
\end{abstract}

be probed and visualized dynamically in situ. ${ }^{5,6}$ Despite being an enabling bioanalytical technique, serious limitations remain for contemporary approaches of molecular imaging with fluorescence. For instance, it is still a challenge to design a probe that can be delivered reliably to particular intracellular organelles in cells of a targeted organ, in which molecular events of interest occur. ${ }^{7}$ Moreover, a difficulty to control, or insufficient knowledge about, the concentration of molecular probes retained at the designated sites might obscure a quantitative deciphering of the information obtained from these approaches.

Distinct from conventional means of molecular imaging that generally involve an administration of an exogenous reporting agent to a human body, herein we propose an alternative approach by using endogenous autofluorescence to examine cellular deoxygenation and reoxygenation. Flavins, comprising

Received: February 16, 2014

Accepted: April 10, 2014

Published: April 10, 2014 


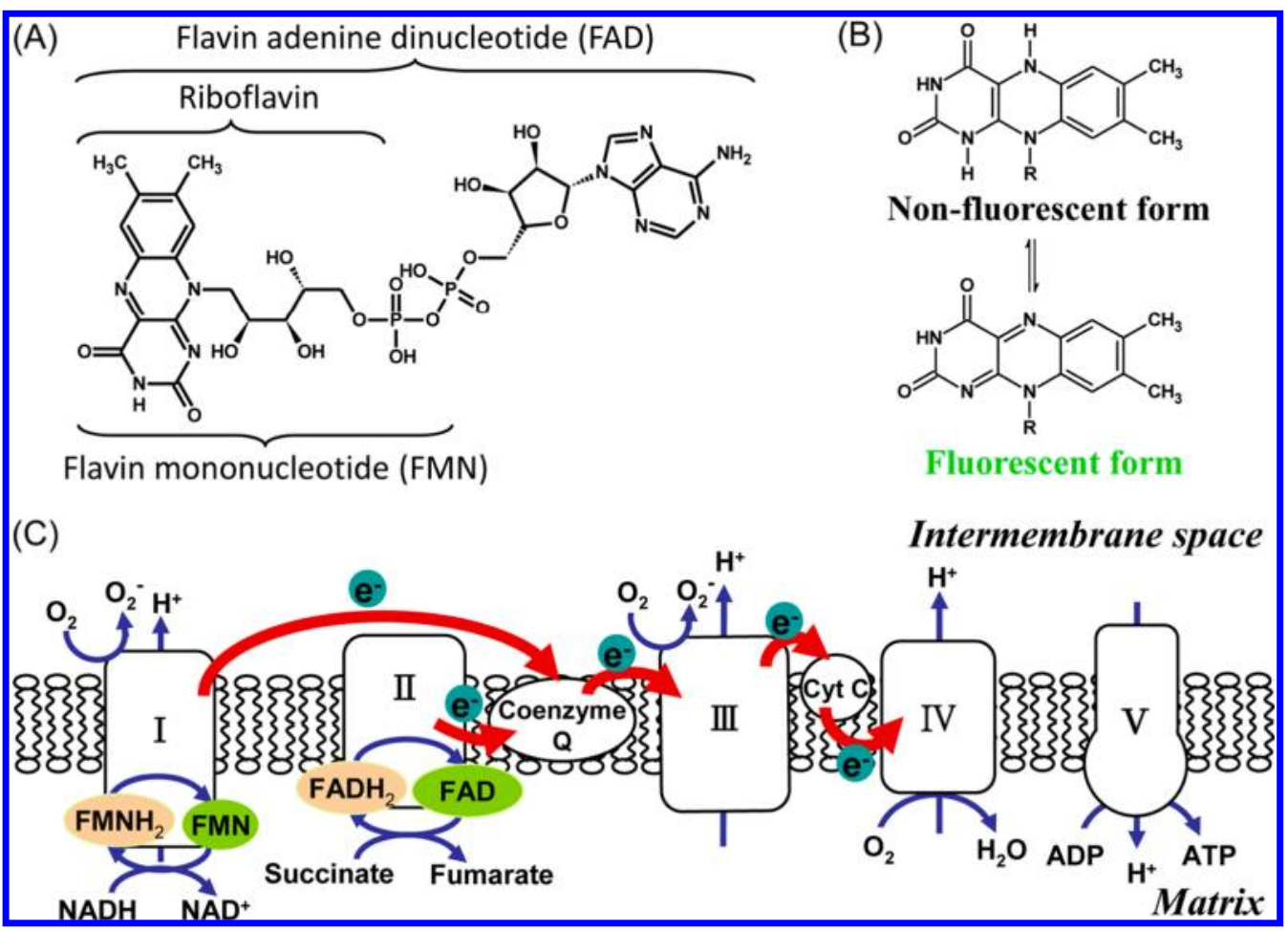

Figure 1. Schematic illustration of the rationale. (A) Structures of common endogenous flavins: flavin adenine dinucleotide (FAD), flavin mononucleotide (FMN), and riboflavin. (B) Illustration showing that the fluorescent FAD (or FMN) becomes nonfluorescent $\mathrm{FADH}_{2}\left(\right.$ or $\mathrm{FMNH}_{2}$ ) after accepting two electrons and two protons. (C) Cartoon showing the critical role of the redox conversion of flavins in mediating the transfer of electrons in the mitochondrial electron-transport chain. I: NADH dehydrogenase; II: succinate dehydrogenase; III: cytochrome c-coenzyme Q oxidoreductase; IV: cytochrome oxidase; V: ATP synthase.

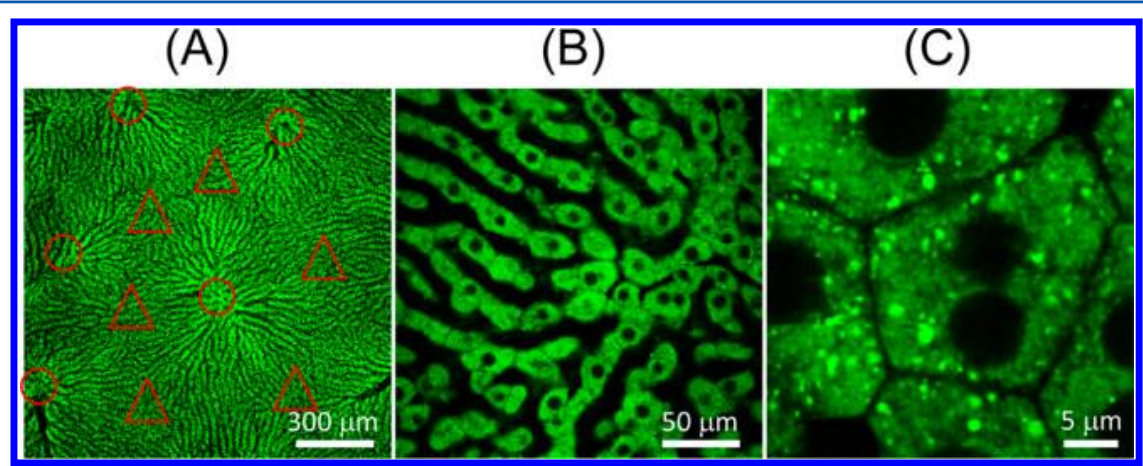

Figure 2. Intravital autofluorescent images of rat liver. (A) Image at small magnification showing characteristic histological features of liver tissues (O: the central vein; $\triangle$ : the portal triad). (B) Image of moderate magnification that clearly resolves individual sinusoids and hepatocytes. (C) Image of large magnification showing individual nuclei and an intercellular junction. These images were obtained with $\lambda_{\mathrm{ex}}=445 \mathrm{~nm}$ and $\lambda_{\mathrm{em}}=458-630$ nm. Scale bars: (A) $300 \mu \mathrm{m}$, (B) $50 \mu \mathrm{m}$, and (C) $5 \mu \mathrm{m}$.

mainly flavin adenine dinucleotide (FAD), flavin mononucleotide (FMN), and riboflavin (Figure 1A), act as redox cofactors in cellular metabolism through acceptance and donation of electrons. ${ }^{9}$ Preceding authors have reported that the cellular autofluorescence of flavins is produced predominantly from mitochondrial flavins that include flavoproteins in the mitochondrial ETC, flavins bound to lipoamide dehydrogenase $(\mathrm{LipDH})$, and other nonspecific flavins. ${ }^{10,11}$ It has been known that they switch between the fluorescent oxidized form and nonfluorescent reduced form (Figure 1B) during the redox process. ${ }^{12}$ As molecular dioxygen is the final electron acceptor along the mitochondrial ETC (Figure 1C), we hypothesize that a depletion of $\mathrm{O}_{2}$ during ischemia would tend to shift the balance of the flavins in the mitochondrial ETC toward the nonfluorescent reduced form, causing a decreased autofluor- escence. The autofluorescent variation of flavoproteins in the mitochondrial ETC could accordingly serve as a sensitive and specific indicator of the deoxygenation and reoxygenation of mitochondria during IR in vivo.

The central objective of this work is to test our hypothesis and to demonstrate the application of autofluorescent molecular imaging as a novel bioanalytical tool to assess IR. Herein, we chose hepatic IR as an example for a proof of principle. We first visualized microscopically a rat liver in vivo with intravital autofluorescent imaging (Figure 2). We then demonstrated delicately that the cellular autofluorescence under our experimental design was originated predominately from mitochondria. The emission spectra of isolated mitochondria, hepatocytes in vitro, and rat liver in vivo all possess a peak around $530 \mathrm{~nm}$, which conforms to the spectral 


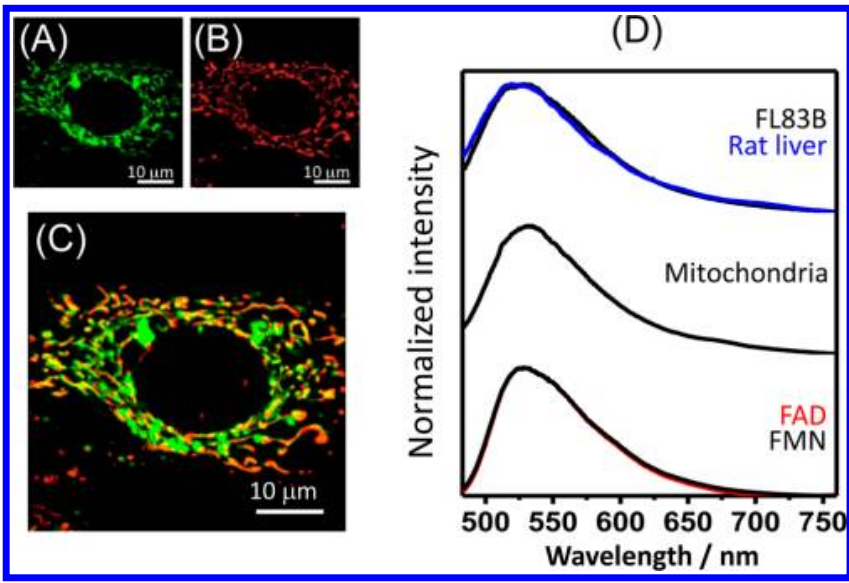

Figure 3. Results of experiments to identify the molecular origin of autofluorescence. (A) Autofluorescent image of a hepatocyte (FL83B) obtained before administration of labeling stains; $\lambda_{\mathrm{ex}}=445 \mathrm{~nm}$ and $\lambda_{\mathrm{em}}$ $=458-630 \mathrm{~nm}$. Scale bar: $10 \mu \mathrm{m}$. (B) Fluorescent image of the same cell obtained after labeling with a stain specific to mitochondria (MitoTracker Red); $\lambda_{\mathrm{ex}}=561 \mathrm{~nm}$ and $\lambda_{\mathrm{em}}>610 \mathrm{~nm}$. (C) Overlay of autofluorescent (green) and fluorescent (red) images. (D) Comparison of the autofluorescent spectra obtained from hepatocytes (FL83B; black), the liver of a living rat (blue), and mitochondria isolated from a rat liver (black) with the fluorescent spectra acquired from flavin adenine dinucleotide (FAD; red) and flavin mononucleotide $(\mathrm{FMN}$; black). $\lambda_{\mathrm{ex}}=445 \mathrm{~nm}$.

characteristics of flavins (Figure 3). Experimental IR in vitro (Figure 4) and in vivo (Figure 5) showed that the cellular autofluorescence intensity decreased around 30\% from baseline immediately after ischemia and resumed progressively to baseline during reperfusion. This ratio of fluorescent decrease is consistent with that of cells with interrupted mitochondrial ETC. The dynamic autofluorescent images of a rat liver revealed further a spatiotemporal correlation with the perfused blood flow during reperfusion (Figure 6). Our approach generates an unprecedented opportunity to interrogate mitochondria, the machinery central to the pathophysiology of $\mathrm{IR}^{13}$ with great spatiotemporal specificity. We expect the core of this work to be translated into a medical device of molecular imaging; its realization would shed new light on clinical diagnostics and therapeutic interventions targeting IR injuries of not only the liver but also other vital organs including the brain and heart.

\section{MATERIALS AND METHODS}

Reagents. Sucrose, Tris, ethylene glycol tetraacetic acid (EGTA), antimycin A (AA), dimethyl sulfoxide (DMSO), flavin adenine dinucleotide (FAD), flavin mononucleotide (FMN), and rhodamine B isothiocyanate-dextran (RITCdextran) $(\mathrm{MM}=70000 \mathrm{u})$ (Sigma-Aldrich), fetal bovine serum, Ham's F12 nutrient mixture, and penicillin/streptomycin mixture (Invitrogen) were purchased from the indicated sources.

Isolation of Mitochondria. To isolate mitochondria, we minced a rat liver and homogenized it with a Dounce homogenizer (Kontes Glass) in an ice-cold buffer (sucrose $250 \mathrm{mM}$, Tris $5 \mathrm{mM}$, EGTA $1 \mathrm{mM}, \mathrm{pH}$ 7.4). The homogenized tissue was centrifuged at $4{ }^{\circ} \mathrm{C}$ at $1000 \mathrm{~g}$ for $10 \mathrm{~min}$ to remove the chunks and twice at $12000 \mathrm{~g}$ for $15 \mathrm{~min}$ to collect mitochondria.

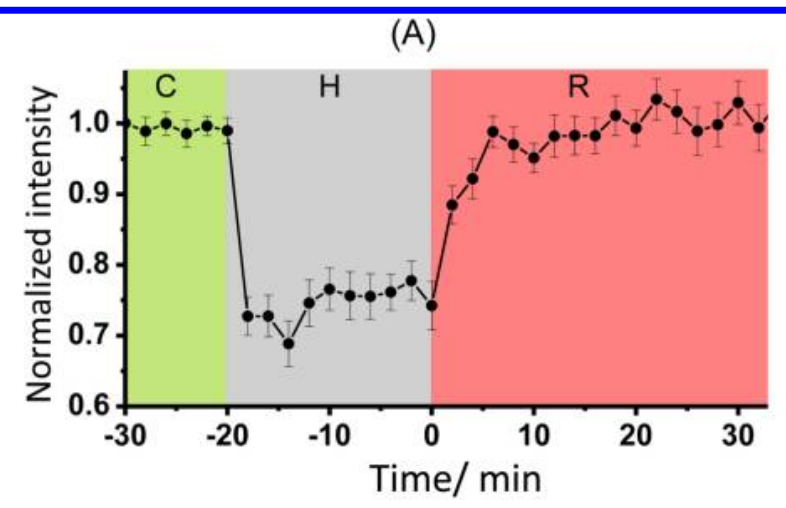

(B)
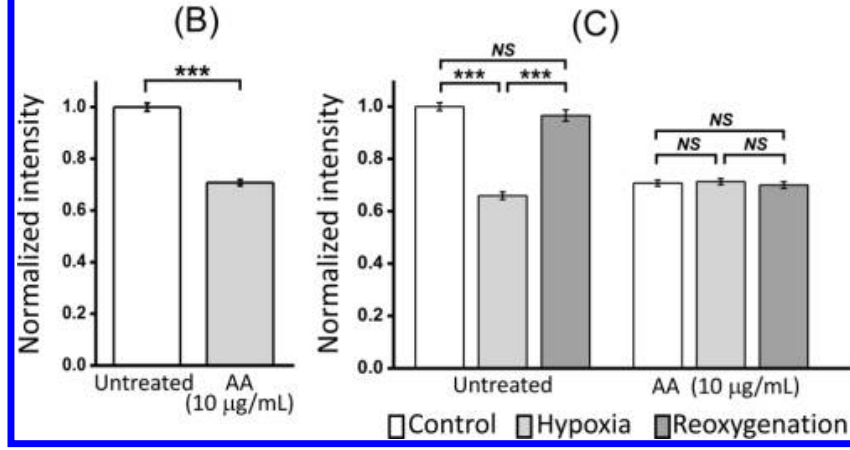

Figure 4. Autofluorescent imaging of cells subject to hypoxia and reoxygenation. (A) Temporal autofluorescent intensity recorded during the temporal course of hypoxia $(20 \mathrm{~min})$ and reperfusion (35 $\mathrm{min}$ ). The data were calculated from 29 cells in total from three independent measurements; the total intensity was normalized relative to the control (before hypoxia). C: control; $\mathrm{H}$ : hypoxia; R: reoxygenation. $\lambda_{\mathrm{ex}}=445 \mathrm{~nm}$ and $\lambda_{\mathrm{em}}=458-630 \mathrm{~nm}$. (B) Comparison of the autofluorescent intensity measured from the control (cells incubated with $0.5 \% \mathrm{DMSO} ; 60 \mathrm{~min}$ ) with that from cells incubated with antimycin A (AA; in 0.5\% DMSO) for the same duration. Each set of data was calculated from 90 cells in total from three independent measurements; the total intensity was normalized relative to the control. *** $p<0.001$. (C) Autofluorescent intensity measured on the control (before hypoxia; white) and after subjection to hypoxia (20 min; light gray) followed with reoxygenation (30 min; dark gray) for cells subject to no pretreatment (cells incubated with $0.5 \%$ DMSO; 60 $\mathrm{min}$ ) or a pretreatment of AA (in $0.5 \% \mathrm{DMSO}$ ) for the same duration. Each set of data was calculated from 90 cells in total from three independent measurements after subtraction of a background; the total intensity was normalized relative to the control corresponding to cells treated with DMSO alone. $* * *: p<0.001 ; N S$ : insignificant.

Culturing of hepatocytes. Mouse hepatocytes (FL83B) were grown in a F12 medium supplemented with thermally inactivated fetal bovine serum (10\%) and a mixture of penicillin and streptomycin $(100 \mathrm{U} / \mathrm{mL})$ at $37{ }^{\circ} \mathrm{C}$ and $5 \% \mathrm{CO}_{2}$ and were split 1:4 every $2 \mathrm{~d}$ after approximately $90 \%$ confluence. Before imaging, the cells were plated in glass-bottomed Petri dishes (MatTek) and cultured for another $2 \mathrm{~d}$.

Inhibitory Assay of the Mitochondrial Electron-Transport Chain. To inhibit the mitochondrial ETC, we incubated cells with AA $(10 \mu \mathrm{g} / \mathrm{mL}$, in $0.5 \%$ DMSO $)$ for $1 \mathrm{~h}^{14,15}$ To prepare a control, we incubated cells with a serum-free F12 medium containing $0.5 \%$ DMSO for the same duration.

Hypoxia and Reoxygenation of Hepatocytes. To facilitate imaging of cells during hypoxia and reoxygenation of cells, we placed the Petri dish that contained cells in a perfusion chamber (Figure S1A of the Supporting Information). We prepared a hypoxic medium on bubbling $\mathrm{N}_{2}$ gas into a serum- 


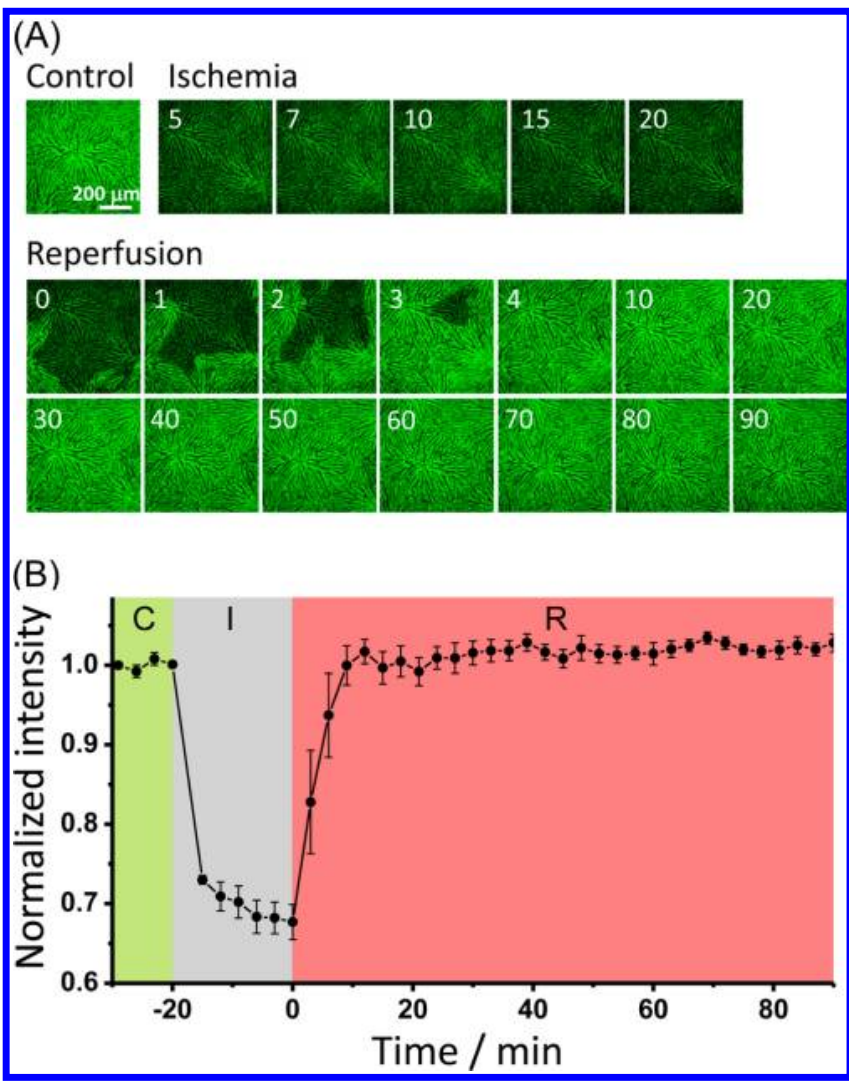

Figure 5. Intravital autofluorescent imaging of rat liver recorded during ischemia and reperfusion. (A) Time-lapse images of a rat liver obtained before ischemia (control), during ischemia (20 min), and reperfusion $(90 \mathrm{~min}) ; \lambda_{\mathrm{ex}}=445 \mathrm{~nm}$ and $\lambda_{\mathrm{em}}=458-630 \mathrm{~nm}$. Scale bar: $200 \mu \mathrm{m}$. (B) Temporal autofluorescent intensity measured during ischemia and reperfusion $(n=5)$. Each data point was calculated from the integrated intensity of an image after subtraction of the background and was normalized relative to the average intensity of the control. C: control; I: ischemia; R: reperfusion.

free F12 medium until the concentration of dissolved $\mathrm{O}_{2}$, determined with an oxygen meter (OM-51, Horiba, Japan), was less than 1\% (Figure S1B of the Supporting Information). To establish a hypoxic condition, we replaced the medium in the Petri dish with a hypoxic medium made to flow with a peristaltic pump; a restoration from the hypoxic condition was achieved on replacing the medium with a normal medium. The sham experiment was performed in the same manner except that a normal medium was used at both stages.

Animals and Feeding Protocols. This work was approved by the Animal Investigation Committee of National Chiao Tung University. Male Wistar rats (BioLASCO Experimental Animal Center, Taiwan) aged between 7 and 9 weeks with a mass of 250-300 g were used for this work. All animals were allowed free access to standard diets and drinking water and were maintained under cycles of light for $12 \mathrm{~h}$ and darkness for $12 \mathrm{~h}$. The animals fasted for $24 \mathrm{~h}$ before experiments.

Hepatic Ischemia and Reperfusion. We anesthetized the animals with an intraperitoneal injection of pentothal $(2.5 \%, 70$ $\mathrm{mg} / \mathrm{kg}$ ) and performed a midline laparotomy to expose the liver. To induce ischemia, the hepatic artery and the portal vein that supplied blood to the median and left lobes of the liver were clamped with a bulldog clamp for a designed duration; to induce reperfusion, the clamp was released from the vessels to restore blood flow (Figure S2A of the Supporting Informa- tion). ${ }^{16}$ For imaging, the animal was placed in a side-lying position on a custom-made sample stage of an inverted optical microscope with the left lobe of the liver facing the objective lens; throughout the experiment, the abdominal cavity of the animal was wrapped with moisturized gauze to prevent drying, and the animal was wrapped with heating pads to maintain body temperature (Figure S2B of the Supporting Information). At the end of the experiment, the rat was euthanized with an injection of excessive pentothal.

Optical Imaging and Emission Spectra. A modified confocal laser-scanning optical microscope (FV300, Olympus, Japan) was employed for optical imaging and spectral measurements. To measure emission spectra, we directed the emission through the back port of the microscope, coupled it into an optical fiber, and then analyzed it with a spectrograph (Shamrock SR-303i, Andor Technology, U.K.). To image with autofluorescence alone, we employed a diode laser $(445 \mathrm{~nm}$, PhoxX445, Omicron, Germany) for excitation and detected the emission between 458 and $630 \mathrm{~nm}$. To obtain fluorescent images of RITC-dextran simultaneously, we merged the light from another diode laser $(561 \mathrm{~nm}$, Cobolt Jive $561 \mathrm{~nm}$, Cobolt, Sweden) with the light from the former laser, and detected the emission simultaneously between 458 and $530 \mathrm{~nm}$ (corresponding to the autofluorescence of flavins) and that greater than $610 \mathrm{~nm}$ (fluorescence of RITC-dextran). Throughout this work, the excitation was set at $10 \mu \mathrm{W}$ to minimize photobleaching while maintaining a satisfactory ratio of signal-to-noise of the images.

Image Analysis. Analysis of images was undertaken with freeware (ImageJ; NIH). For images of cells, we first set the region within a cell, excluding the nucleus as a region of interest (ROI). We then integrated the intensity of all pixels in the ROI and subtracted a background that was determined on setting a ROI of the same area in the medium. The result was then normalized relative to the corresponding control. For images of a rat liver, we integrated the intensity of all pixels in an image and subtracted a background that was determined from a region of the blood vessel. The result was then normalized relative to the corresponding control. To take photobleaching into account, we calibrated the temporal autofluorescent intensity shown in this work with a single-exponential function (see Figure S3 of the Supporting Information and the captions for detail).

Statistical Methods. All data are expressed as mean \pm SEM. We compared the means of two groups using the twotailed Student's t test. The levels of statistical significance were set at $* p<0.05$, ** $p<0.01$, and $* * * p<0.001$, respectively.

\section{RESULTS}

Demonstration of Microscopic Imaging of Rat Liver in vivo with Autofluorescence. We first tested whether it is feasible to visualize histological details of a rat liver in vivo with autofluorescence that was excited near the absorption maximum of flavins $\left(\lambda_{\mathrm{ex}}=445 \mathrm{~nm}\right)$. The images at a small magnification (Figure 2A) exhibited distinct histological characteristics of a liver, showing clearly the parenchyma, central vein, portal triad, and sinusoids. As shown from the magnified images (Figure 2, panels $B$ and $C$ ), the autofluorescence arose mainly from the hepatocytes; in contrast, the signal produced from the nuclei, the gap junction between hepatocytes, the circulating blood cells, and the plasma in the sinusoid was slight. 


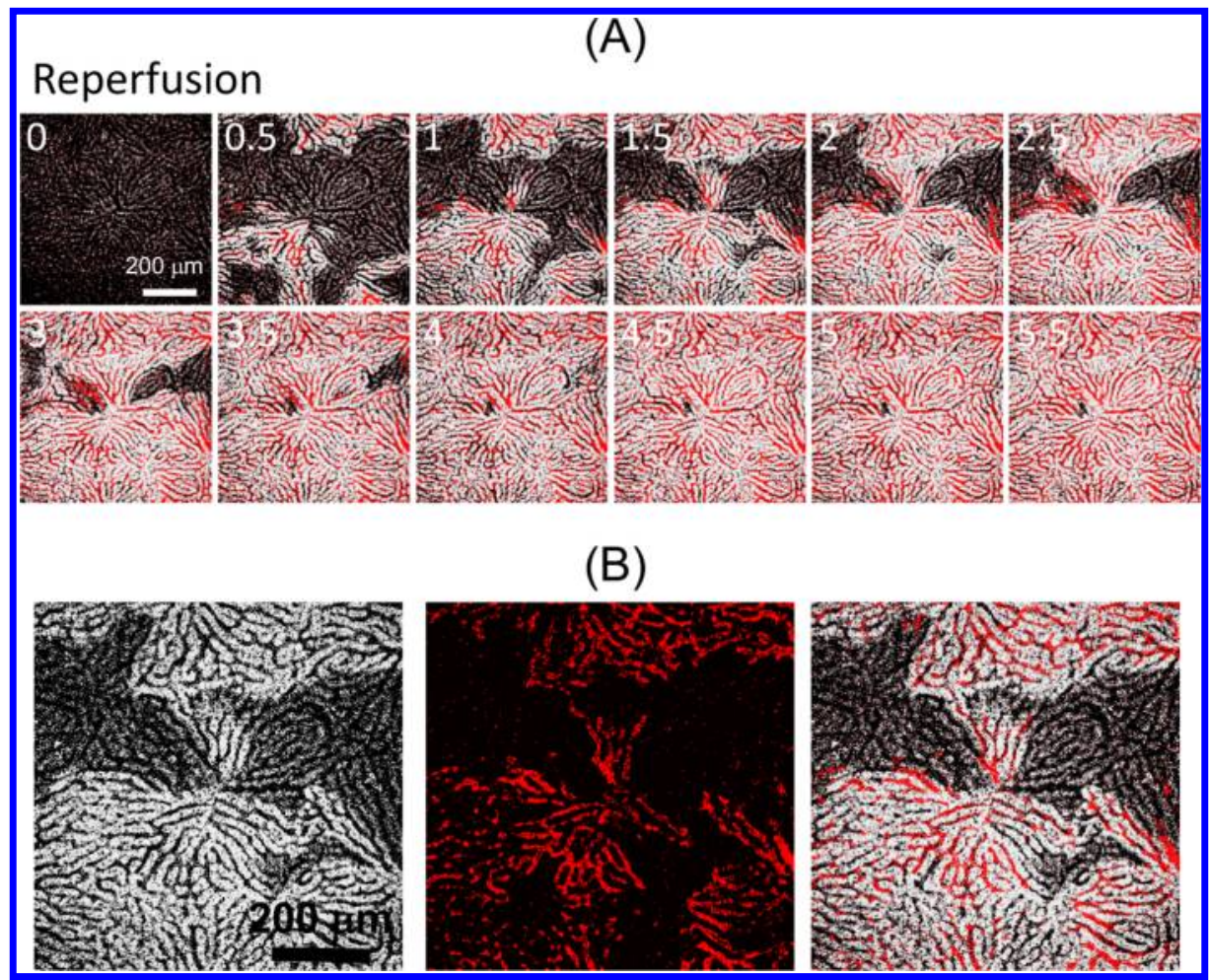

Figure 6. Spatiotemporal relation between the autofluorescent images and the fluorescent images of the stained blood flow. (A) Overlaid time-lapse images obtained during reperfusion. Red: fluorescence $\left(\lambda_{\mathrm{ex}}=561 \mathrm{~nm}\right.$ and $\left.\lambda_{\mathrm{em}}>610 \mathrm{~nm}\right)$; gray: autofluorescence $\left(\lambda_{\mathrm{ex}}=445 \mathrm{~nm}\right.$ and $\lambda_{\mathrm{em}}=458-530$ $\mathrm{nm}$ ). Scale bar: $200 \mu \mathrm{m}$. (B) A representative set of simultaneously acquired images obtained 1.5 min after reperfusion (left: autofluorescence; middle: fluorescence; right: overlaid).

These results demonstrate that the autofluorescence excited near the maximal absorption of flavins enabled microscopic visualization of a rat liver in vivo. In particular, the great contrast of the autofluorescent images rendered individual hepatocytes and the hepatic sinusoidal structure discernible in vivo without an exogenous labeling stain.

Identification of the Molecular Origin of Cellular Autofluorescence. To aid identification of the molecular origin of autofluorescence detected in the animal experiments, we characterized the autofluorescence produced from cultured hepatocytes (FL83B cells) with various tests.

We first imaged cells with the same excitation employed in the animal experiments. The result showed that the autofluorescent images of cells exhibited features resembling the morphology of mitochondria (Figure 3A). To verify that the autofluorescence was associated with mitochondria, we labeled the cells with a stain specific to mitochondria (MitoTracker Red $)^{17}$ and acquired an image with the fluorescence of the stains (Figure 3B). It is remarkable that the fluorescent (red) and autofluorescent (green) images obtained on the same cell colocalized extensively (Figure 3C). This result indicates that the autofluorescence was produced, to a large extent, from mitochondria. Despite the extensive colocalization, we noted also that some bright autofluorescent spots failed to colocalize with the fluorescence of MitoTracker Red; we return to this point in Discussion. We proceeded to characterize the spectral profile of the autofluorescence. The emission spectra measured from cultured hepatocytes, rat liver in vivo and mitochondria isolated from rat liver, appeared similar; more importantly, they resembled that measured from flavins (Figure 3D).
These collective results indicate strongly that the autofluorescence of cultured hepatocytes or rat liver excited with a laser at $\lambda_{\text {ex }}=445 \mathrm{~nm}$ was produced mainly from flavin molecules residing in mitochondria.

Attribution of the Kinetic Variation of Cellular Autofluorescence to the Altered Balance between the Mitochondrial Flavins in Oxidized and Reduced Forms during Hypoxia and Reoxygenation. As described in the introduction, we hypothesized that ischemia and reperfusion might alter the balance between the flavoproteins in oxidized and reduced forms residing in the mitochondrial ETC, leading to a kinetic variation of the cellular autofluorescence.

To test this hypothesis, we measured the temporal autofluorescence of cells undergoing hypoxia and reoxygenation in vitro, according to a protocol described in the experimental section (see also Figure S1 of the Supporting Information). The result showed that the cellular autofluorescence decreased by approximately $27 \%$ from the baseline immediately after we replaced the medium of cells with a hypoxic medium, and restored gradually to the baseline after the cells were perfused with a normoxic medium (Figure 4A). In contrast, the autofluorescence of cells that underwent a sham experiment exhibited no discernible change (data not shown). The strong correlation of the cellular autofluorescence with the hypoxiareoxygenation stages is remarkable and is consistent with our hypothesis.

We proceeded to verify whether the kinetic variation of the autofluorescence during stages of hypoxia and reoxygenation linked mechanistically to the deoxygenation and reoxygenation of flavoproteins in the mitochondrial ETC, rather than resulting from other mechanisms unrelated to the mitochondrial ETC. In an experiment on a negative control, we tested whether an 
interruption of the mitochondrial ETC with a chemical means resulted in decreased autofluorescence similar to what we observed in the stage of hypoxia. We employed AA that binds to cytochrome $c$ reductase to disrupt the electron flow of the mitochondrial ETC. The result shows that cellular autofluorescence decreased significantly (29\%) after a treatment of cells with AA (Figure 4B). Moreover, the extent of the decrease in the cellular autofluorescence after the treatment of AA was comparable with that observed during hypoxia (29\% vs $27 \%$ ). This quantitative agreement between the two independent experiments is remarkable and indicates strongly that the variation of the autofluorescence observed in these experiments resulted from a common molecular origin (i.e., the flavins in the mitochondrial ETC).

To test this deduction more rigorously, we pretreated cells with AA to disrupt the mitochondrial ETC and recorded the autofluorescence of the cells subject to hypoxia and reoxygenation in a way similar to the preceding experiment. In sharp contrast to the distinct autofluorescent kinetics observed from the sham experiment but conforming to the expected inhibitory activity of AA on the mitochondrial ETC, the autofluorescence of the pretreated cells altered negligibly during hypoxia and reoxygenation (Figure 4C).

In accordance with these multiple lines of experimental evidence, we conclude that the kinetic variation of the autofluorescence during hypoxia-reoxygenation resulted directly from the altered balance between the flavoproteins in oxidized and reduced forms residing in the mitochondrial ETC. This conclusion supports our fundamental hypothesis. More importantly, it produces a possibility to specifically interrogate the deoxygenation and reoxygenation of mitochondria during IR with autofluorescence of mitochondrial flavoproteins. We proceeded accordingly to translate this finding to image hepatic IR in vivo.

Demonstration of Molecular Imaging of Hepatic IR in Vivo with the Autofluorescence of Mitochondrial Flavins. We established IR on the rat liver according to a protocol described in the experimental section (see also Figure S2 of the Supporting Information). We noted that the autofluorescent images of the rat liver turned dim relative to the control (before ischemia) soon after the blood vessels were clamped and remained so dim throughout the following temporal course of ischemia (in total $20 \mathrm{~min}$ ); in contrast, the autofluorescent intensity restored progressively after the clamp was released (Figure 5A; see also Video 1 (ac5006469_si 002.avi) of the Supporting Information).

The kinetic variation of the autofluorescence during IR was consistently observed for the five rats examined in this test. As revealed from a quantitative analysis of the temporal images (Figure 5B), the autofluorescent intensity remained stable before the blood vessels were clamped (the basal value). The autofluorescence declined greatly soon after a cessation of blood flow comprising $84 \%$ of the total attenuation within the first $5 \mathrm{~min}$ ( $27 \%$ of total decrease $32 \%$ relative to the baseline), but the temporal decrease became moderate after this initial large decline. When the clamp was removed to have the blood flow resume, the autofluorescence restored gradually, taking about $6 \mathrm{~min}$ to recover to approximately $93 \%$ of the basal value ( 9 min to $99 \%$ of the baseline), and remained at a stable level thereafter. These results show clearly that the kinetic variation of the autofluorescence correlated strongly with the stages of IR.
Spatiotemporal Correlation between the Reoxygenation of Mitochondria and the Restoration of Microcirculatory Blood Flow during Reperfusion. In the preceding experiment, we noted a distinct spatiotemporal pattern of the autofluorescent images during the temporal course of reperfusion. Specifically, the recovery of the autofluorescence was temporally prolonged (delay $6 \mathrm{~min}$ from the removal of the clamp to a restoration to $93 \%$ of the baseline) and spatially heterogeneous during reperfusion [Figure 5A; see also Video 1 (ac5006469_si_002.avi)]. Consistent with our hypothesis, we attribute the recovery of the autofluorescence of mitochondrial flavoproteins to the restoration of hepatic microcirculation.

To reveal the spatiotemporal correlation between mitochondrial reoxygenation and hepatic microcirculation, we injected RITC-dextran intravenously to stain the blood flow before the release of the clamp, and recorded simultaneously the autofluorescent images and the fluorescent images of RITCdextran. Overlay of the two sets of temporal images that were acquired simultaneously on the same hepatic region during reperfusion showed clearly that the recovery of the autofluorescence coincided largely with the resumption of the fluorescence of RITC-dextran (gray: autofluorescence; red: fluorescence of RITC-dextran; Figure 6 and Video 2 (ac5006469_si_003.avi) of the Supporting Information). In particular, the hepatocytes that were adjacent to the sinusoids with restored microcirculation (highlighted in red) exhibited intense autofluorescence (highlighted in gray), whereas the cellular autofluorescence remained consistently dim for the hepatocytes near the sinusoids in which the microcirculation did not resume.

This result demonstrated that the reoxygenation of mitochondria correlated with the restoration of hepatic microcirculation during reperfusion (after ischemia for 20 $\min$ ). Moreover, it indicates that the autofluorescence, being switched off and on, can reveal not only the deoxygenation and reoxygenation of mitochondria but also the cessation and resumption of microcirculation.

\section{DISCUSSION}

IR injury is the key pathological element of myocardial infarction, stroke, shock, cardiac arrest, vascular surgery, and organ transplant and is recognized to be responsible for the complications of these critical clinical events. ${ }^{1}$ In this work, we demonstrate molecular imaging of hepatic IR with the autofluorescence of endogenous flavins. In comparison with molecular imaging in conventional modalities that generally require an administration of exogenous reagents, the utility of autofluorescence entirely eliminates the need of exogenous reporters, and the limitations and drawbacks consequently associated with these reporters such as cytotoxicity. ${ }^{18}$ From a practical perspective, the core finding of this work can be prospectively translated toward the development of a medical device for clinical applications. Although our work focused on hepatic IR, we expect the same approach to be extensible to an investigation of IR occurring in other organs such as cerebral stroke in the brain, acute coronary events in the heart, or transplant of visceral organs such as kidney or liver. For the latter, our approach should be useful not only for assessing organs before donation but also for evaluating the viability of organs during and after transplantation. As our approach allows a direct visualization of the deoxygenation and reoxygenation of cells and a revelation of microcirculatory blood flow in vivo, an 
application of our approach to assess wound healing ${ }^{19}$ or the vascular biology of cancer ${ }^{20}$ is also expected.

Although numerous authors have reported the application of autofluorescence to probe tissues or organs subject to $\mathrm{IR}^{21-23}$ in these preceding works was employed almost exclusively the autofluorescence of nicotinamide adenine dinucleotide $(\mathrm{NADH})$ alone or in combination with FAD; mostly a temporal intensity at a single point of the tissues or images at small magnification was reported. Our work provides the first demonstration of the specific utility of flavins that reside in the mitochondrial ETC for molecular imaging of IR at high resolution. Mitochondria are particularly vulnerable, and their damage might contribute to the cellular damage during IR; ${ }^{13}$ the excessive generation of detrimental reactive oxygen species mediating the mitochondrial ETC has been reported to be responsible for the pathogenesis of reperfusion injury. ${ }^{24,25} \mathrm{An}$ ability to interrogate mitochondria with molecular specificity is hence of fundamental importance. Through molecular imaging at high resolution, we resolved the autofluorescent variation of a liver at a cellular level in vivo and discovered a strong correlation between the autofluorescent kinetics and the cellular and microcirculatory changes during each stage of IR.

As described, the autofluorescence of $\mathrm{NADH}$ to probe the metabolic status of cells or organism is employed more commonly because of the great abundance and large quantum yield of NADH and has yielded significant discoveries. ${ }^{12,26-28}$ Among its limitations or drawbacks, as $\mathrm{NADH}$ distributes abundantly in both cytosol and mitochondria, it is difficult to separate a contribution from the cytosolic and mitochondrial pyridine nucleotides, making the autofluorescence of pyridine nucleotides less specific to mitochondria. In contrast, the autofluorescence of flavins is dominated by flavins residing in mitochondria. ${ }^{29}$ More importantly, we show that the autofluorescent variation during IR is a direct manifestation of the deoxygenation and reoxygenation of mitochondria and, more specifically, the altered balance of flavins in oxidized and reduced forms that reside in the mitochondrial ETC. These unique features make the autofluorescence of flavins a particularly attractive endogenous reporter of molecular imaging of IR given the important pathophysiological role of mitochondria in IR. With regard to the excitation employed to produce autofluorescence, the utility of flavins offers another advantage relative to pyridine nucleotides. The absorption maximum of pyridine nucleotides occurs about $350 \mathrm{~nm}^{26,30}$ In addition to a requirement to use a more costly ultraviolet (UV) laser for excitation, an exposure of tissues to UV light inevitably causes photochemical damage to cells or tissues, especially when a prolonged exposure and long-term monitoring are needed. Moreover, the utility of UV excitation for pyridine nucleotides increases the probability to excite simultaneously endogenous molecules of various types, and obscures the interpretation of the results. In contrast, flavins can be excited effectively with visible light, which decreases not only the probability of photochemical damage but also the chance to excite multiple endogenous fluorophores.

We do not exclude a contribution of other endogenous fluorophores to the autofluorescence observed in our experiments. As described, we consistently observed an autofluorescence decreased approximately $30 \%$ in the hypoxic stage of cells in vitro (Figure 4A) or the ischemic stage of a rat liver in vivo (Figures $5 \mathrm{~B}$ ); we attribute this decreased autofluorescence to a shift of the balance toward the reduced form of the flavins in the mitochondrial ETC. Consistent with this deduction, a pretreatment of an inhibitor of mitochondrial ETC (AA) to cells yielded a decreased autofluorescence (Figure $4 B$ ), and the autofluorescent variation became negligible during the hypoxic stage (Figure 4C). We hence suggest that the $70 \%$ background observed during ischemia (or hypoxia) or after the treatment with AA comprise contributions mainly from other mitochondrial flavins not associated with mitochondrial ETC, such as flavins bound to lipoamide dehydrogenase ( $\mathrm{LipDH})$, cytosolic flavins, or residual flavins in the mitochondrial ETC that remain in oxidized forms during ischemia/hypoxia. Other endogenous fluorophores such as cytosolic lipofuscin that are excited with blue light might contribute also to this background. ${ }^{31,32}$ Consistent with the latter condition, we noted that the autofluorescent images of cultured hepatocytes included some bright spots that were not colocalized with the morphological feature of mitochondria highlighted with the stains specific to mitochondria (Figure 3C); the spectrum measured from these bright spots was distinct from that obtained from flavins (Figure S4 of the Supporting Information vs Figure 3D), exhibiting a long tail on the red shoulder.

A common concern about application of a single-photon fluorescent microscope for imaging in vivo is the limited penetration of excitation into biological tissues. The intravital autofluorescent images observed in this work were obtained from cells that were located immediately beneath the capsule of the rat liver. Despite this limitation, we contend that our results are representative, given that the liver consists of repeated functional units called lobules (see also Figure 2A). ${ }^{33}$ Although a two-photon microscope that utilizes a near-infrared pulsed laser can increase the penetration depth, ${ }^{34}$ such a laser is expensive and beyond access by many investigators. The inherent technical issues associated with the delivery of ultrashort laser pulses in an optical fiber (such as the stretching of pulses in the highly dispersive optical fiber and the chromaticity between the excitation and the emission) $)^{35}$ might further hinder the translation of a multiphoton microscope into a clinical imaging device.

\section{CONCLUSIONS}

We have demonstrated autofluorescent molecular imaging that varied with the stages of IR and exhibited a great spatiotemporal specificity to mitochondria without employing exogenous reporting agents. We elucidated, in particular, the molecular origin of the autofluorescent variation observed during IR, and showed that the autofluorescent resumption correlates strongly with the restoration of microcirculatory blood flow during reperfusion. Our approach opens a new route to investigate IR and might prospectively become a platform of an imaging instrument to target clinical applications such as intraoperative and postoperative diagnosis of organs. An extension of this approach to other pathophysiological processes that involve cellular deoxygenation-reoxygenation is expected.

\section{ASSOCIATED CONTENT}

Supporting Information

Additional information as noted in the text. This material is available free of charge via the Internet at http://pubs.acs.org.

\section{AUTHOR INFORMATION}

\section{Corresponding Author}

*E-mail: ianliau@mail.nctu.edu.tw. 


\section{Author Contributions}

${ }^{\S}$ H.-H.L. and Y.-M.W. have contributed equally to this work.

Notes

The authors declare no competing financial interest.

\section{ACKNOWLEDGMENTS}

We thank Professors Yuan-Pern Lee and Yaw-Kuen Li (National Chiao Tung University) for generous support and Mr. Wei-Ti Wang (NCTU) for technical assistance in animal experiments. National Chiao Tung University, Ministry of Science and Technology, and the MOE-ATU program of Taiwan provided support to I.L.

\section{REFERENCES}

(1) Eltzschig, H. K.; Eckle, T. Nat. Med. 2011, 17, 1391-1401.

(2) Davidson, B. P.; Kaufmann, B. A.; Belcik, J. T.; Xie, A.; Qi, Y.; Lindner, J. R. I. Am. Coll. Cardiol. 2012, 60, 1690-1697.

(3) Kim, C.; Favazza, C.; Wang, L. V. Chem. Rev. 2010, 110, 27562782.

(4) Ntziachristos, V. Annu. Rev. Biomed. Eng. 2006, 8, 1-33.

(5) Thorling, C. A.; Liu, X.; Burczynski, F. J.; Fletcher, L. M.; Roberts, M. S.; Sanchez, W. Y. I. Biomed. Opt. 2013, 18, 101306.

(6) Wang, Y.; Yamamoto, S.; Miyakawa, A.; Sakurai, T.; Ibaraki, K.; Terakawa, S. Neurosurgery 2010, 67, 118-127.

(7) Kobayashi, H.; Ogawa, M.; Alford, R.; Choyke, P. L.; Urano, Y. Chem. Rev. 2010, 110, 2620-2640.

(8) Yang, Y. C.; Lu, H. H.; Wang, W. T.; Liau, I. Anal. Chem. 2011, 83, 8267-8272.

(9) Cosnier, S.; Fontecave, M.; Limosin, D.; Niviere, V. Anal. Chem. 1997, 69, 3095-3099.

(10) Hassinen, I.; Chance, B. Biochem. Biophys. Res. Commun. 1968, 31, 895-900.

(11) Kunz, W. S.; Kunz, W. Biochim. Biophvs. Acta 1985, 841, 237246.

(12) Huang, S. H.; Heikal, A. A.; Webb, W. W. Biophys. J. 2002, 82, $2811-2825$.

(13) Walters, A. M.; Porter, G. A., Jr.; Brookes, P. S. Circ. Res. 2012, $111,1222-1236$

(14) Rieske, J. S.; Lipton, S. H.; Baum, H.; Silman, H. I. J. Biol. Chem. 1967, 242, 4888-4896.

(15) Meany, D. L.; Thompson, L.; Arriaga, E. A. Anal. Chem. 2007, $79,4588-4594$.

(16) Abe, Y.; Hines, I. N.; Zibari, G.; Pavlick, K.; Gray, L.; Kitagawa, Y.; Grisham, M. B. Free Radical Biol. Med. 2009, 46, 1-7.

(17) Dong, X. H.; Heo, C. H.; Chen, S. Y.; Kim, H. M.; Liu, Z. H. Anal. Chem. 2014, 86, 308-311.

(18) Alford, R.; Simpson, H. M.; Duberman, J.; Hill, G. C.; Ogawa, M.; Regino, C.; Kobayashi, H.; Choyke, P. L. Mol. Imaging 2009, 8, 341-354.

(19) Gottrup, F. World J. Surg. 2004, 28, 312-315.

(20) Hoeben, A.; Landuyt, B.; Highley, M. S.; Wildiers, H.; Van Oosterom, A. T.; De Bruijn, E. A. Pharmacol. Rev. 2004, 56, 549-580.

(21) Raman, R. N.; Pivetti, C. D.; Matthews, D. L.; Troppmann, C.; Demos, S. G. Opt. Express 2008, 16, 4930-4944.

(22) Fitzgerald, J. T.; Michalopoulou, A.; Pivetti, C. D.; Raman, R. N.; Troppmann, C.; Demos, S. G. I. Biomed. Opt. 2005, 10, 044018.

(23) Thorling, C. A.; Liu, X.; Burczynski, F. J.; Fletcher, L. M.; Gobe,

G. C.; Roberts, M. S. I. Biomed. Opt. 2011, 16, 116011.

(24) Chen, Y.-R.; Zweier, J. L. Circ. Res. 2014, 114, 524-537.

(25) Tsourkas, A.; Newton, G.; Perez, J. M.; Basilion, J. P.; Weissleder, R. Anal. Chem. 2005, 77, 2862-2867.

(26) Eto, K.; Tsubamoto, Y.; Terauchi, Y.; Sugiyama, T.; Kishimoto, T.; Takahashi, N.; Yamauchi, N.; Kubota, N.; Murayama, S.; Aizawa, S.; Akanuma, Y.; Aizawa, S.; Kasai, H.; Yazaki, Y.; Kadowaki, T. Science 1999, 283, 981-985.
(27) Skala, M. C.; Riching, K. M.; Gendron-Fitzpatrick, A.; Eickhoff, J.; Eliceiri, K. W.; White, J. G.; Ramanujam, N. Proc. Natl. Acad. Sci. U.S.A. 2007, 104, 19494-19499.

(28) Niesner, R.; Peker, B.; Schlusche, P.; Gericke, K. H. ChemPhusChem 2004, 5, 1141-1149.

(29) Koke, J. R.; Wylie, W.; Wills, M. Cvtobios 1981, 32, 139-145.

(30) Villette, S.; Pigaglio-Deshayes, S.; Vever-Bizet, C.; Validire, P.; Bourg-Heckly, G. Photochem. Photobiol. Sci. 2006, 5, 483-492.

(31) Richards-Kortum, R.; Sevick-Muraca, E. Annu. Rev. Phys. Chem. 1996, 47, 555-606.

(32) Marmorstein, A. D.; Marmorstein, L. Y.; Sakaguchi, H.; Hollyfield, J. G. Invest. Ophthalmol. Visual Sci. 2002, 43, 2435-2441.

(33) Rappaport, A. M.; Borowy, Z. J.; Lougheed, W. M.; Lotto, W. N. Anat. Rec. 1954, 119, 11-33.

(34) Helmchen, F.; Denk, W. Nat. Methods 2005, 2, 932-940.

(35) Pawley, J. Handbook of Biological Confocal Microscopy; Springer: New York, 2010. 